\title{
A Functional Approach to Sex: Reproduction in the Créatúr Framework
}

\author{
Amy de Buitléir $\left.{ }^{1(}\right)$, Mark Daly ${ }^{1}$, Michael Russell ${ }^{1}$, and Daniel Heffernan ${ }^{2}$ \\ 1 Athlone Institute of Technology, Athlone, Ireland \\ amy@nualeargais.ie, \{mdaly, mrussell\}@ait.ie \\ ${ }^{2}$ National University of Ireland, Maynooth, Ireland \\ dmh@thphys.nuim.ie
}

\begin{abstract}
Implementing genetics and reproduction for artificial life involves a set of tasks that are only loosely dependent on the type of agent or the method of reproduction. Créatúr is a software framework for automating experiments with artificial life, and a library of modules that can be used to implement agents. In this paper we describe how Créatúr uses Haskell features such as monads, domain-specific embedded languages, and datatype-generic programming to simplify the implementation of genetics and reproduction. We discuss the possibility that type families could support duplicate instance declarations in appropriate circumstances.
\end{abstract}

Keywords: Haskell · Artificial life • Datatype generic programming • Domain specific language

\section{Introduction}

Artificial life (ALife) is a field which attempts to create life-like behaviour using software, hardware, biochemistry or other media; in this paper we focus on software. Whereas biology is the study of "life-as-we-know-it", ALife is the study of "life-as-it-could-be"[1]. ALife is not only used as a simplified model of biological life and ecosystems; it is also increasingly applied to real-world problems as diverse as data mining[2], music composition[3], and management of dam operations in multi-reservoir river systems[4].

The recipe for evolution is simple; the ingredients are[5]:

1. variation: a continuing abundance of different elements,

2. heredity or replication: the capacity to create copies of elements, and

3. differential fitness: the number of copies created depends on the interaction between the features of an element with features of the environment.

All of the complexity and variation of biological life arises from this mechanism, even though "the only thing that changes in evolution is the genes" $[6]$. 
Although the process of evolution is normally associated with biological organisms, it can occur with any substrate as long as those three conditions are met. Hence, evolution is often used in ALife.

To explore how evolution of ALife might be implemented, consider the agent below.

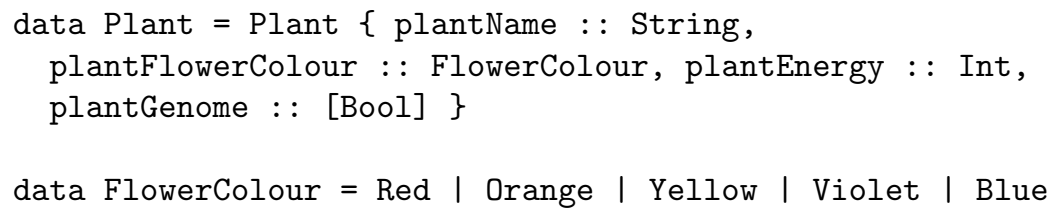

This is of course a very simple example. There is only one genetic trait, plantFlowerColour; it is specified by the plantGenome, which is encoded as a sequence of Bools. (The field plantEnergy is not genetic; it is set to the same initial value for all Plants at "birth".)

Our Plant type has only one strand of genetic material; this illustrates a common approach[7, p. 10f] in evolutionary computation that we will refer to as simplified sexual reproduction. During reproduction, the strands from two parents are recombined to produce two new strands. Two offspring can be created from the new strands. Alternatively, one strand may be chosen at random to create a child, and the other strand discarded. In either case, each parent contributes approximately half of its genetic information to the offspring.

Compare the definition of Plant with the following definition. This agent, called Bug, uses an approach that more closely models sexual reproduction in biology.

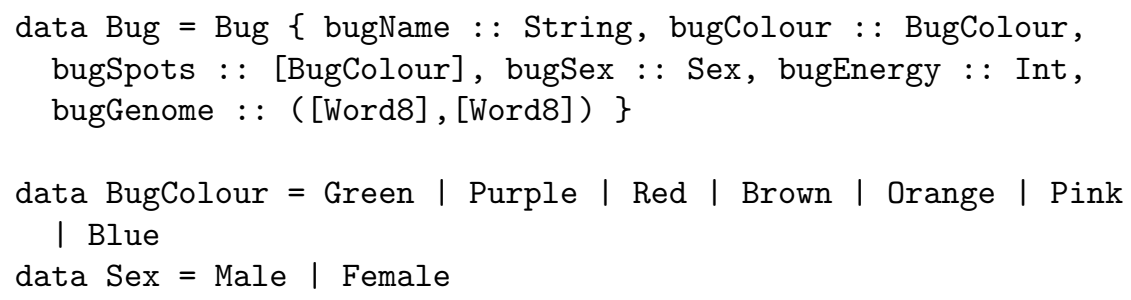

In this case, there are two strands of genetic information, represented by a tuple containing two sequences of Word8s. During reproduction, the two strands from one parent are recombined to produce two new strands. One of those strands is chosen at random to become that parent's contribution to the child's genome. This is analogous to the production of a gamete (ovum or sperm) in biology. The process is repeated for the other parent. Thus the child has two strands of genetic information, one contributed by each parent. As before, each parent contributes approximately half of its genetic information to the offspring.

Although there are differences in the details, the task of implementing either style of reproduction is very similar. The programmer must design a genome, implement recombination of genetic information, support occasional mutation of 
genes, provide a means to encode a set of traits into a strand of genetic information, provide a means to decode strands of genetic information to determine the corresponding traits, and implement the construction of an agent (or solution) from the genome.

The researcher may not care about the precise design of the genome, or its implementation, only requiring that it behaves in a way that supports evolution. Specifically, the genome and the recombination technique must be designed to ensure that offspring are similar to their parents (except in the case of mutation). A straightforward conversion of numeric values to binary is not a good approach; an agent with, say, 18 legs (10010) and one with 20 legs (10100) could produce a child with 31 legs (11111) - not very similar to either parent!

So designing, implementing, and testing a genome is not trivial. Are there tools that can make this easier? As part of our research using ALife to extract knowledge from large data sets with minimal preparation or ramp-up time[8], we work with a variety of agents. We developed Créatúr ${ }^{1}$, which is both a software framework for automating experiments with ALife and a library of modules that can be used (with or without the framework) to implement agents. We chose to implement Créatúr in Haskell based on our positive experience using it to create a neural network[9]. In this paper we describe how Créatúr uses Haskell features such as monads, domain-specific embedded languages, and datatypegeneric programming to address genetics and reproduction. The full source code for Créatúr is available on GitHub[10]; a tutorial is also provided[11].

\section{Datatype-Generic Programming}

Generic programming is programming that references types to be specified later. The actual implementation is automatically generated when the types are finally specified. The Haskell 98 standard[12] includes some support for generic programming, in the form of derived instances, but only for six typeclasses (Eq, Ord, Enum, Bounded, Show and Read). The Glasgow Haskell Compiler (GHC) provided some extensions (Data, Typeable, Functor, Foldable, and Traversable) as part of the Scrap Your Boilerplate system[13-15].

GHC version 7.2 added support for datatype-generic programming as proposed by Magalhães et al. [16]. This lightweight and portable approach allows the programmer to specify how to derive arbitrary class instances. The key is that the "generic" type is represented at runtime using a sum-of-products representation, which involves the following types:

- U1 Unit, used for constructors without arguments

- K1 Constants, additional parameters and recursion of kind *

- M1 Meta-information (constructor names, etc.)

- :+: Sum, which encodes choices between constructors

- :*: Product, which encodes multiple arguments to constructors

${ }^{1}$ Créatúr (pronounced kray-toor) is an Irish word meaning animal, creature, or unfortunate person. 
As a result of this approach, the programmer usually only needs to write implementations for a set of base types, plus an implementation for each of the representation types above. Finally, the end user simply declares their type to be an instance of the desired type (using the DeriveGeneric pragma). We will show an example of this in Section 3.

\section{Gene Encoding}

The Créatúr library provides tools to develop an encoding scheme for a gene or an entire organism. The Genetic class provides the functions for encoding and decoding. Initially we defined the Genetic class using type families, as shown below. The function put writes a gene to a sequence; get reads the next gene in a sequence. The type Sequence represents an encoded gene sequence, for example, [Bool] or [Word8].

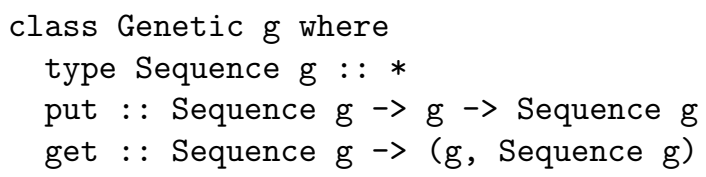

Suppose we want to support a gene sequence type of [Bool]. We would create type instances of Genetic for each Haskell base type that we want to support, such as Char.

instance Genetic Char where type Sequence Char $=[$ Bool $] \ldots$

We would also create instances for U1, K1, M1, :+: and :*:, as discussed in Section 2. A user will then be able to build new types using the supported base types, and declare them to be instances of Genetic. For example,

data MyType = MyType Char $\ldots$ deriving Generic instance Genetic MyType

A problem arises when we want to support multiple types of gene sequences for the base types. For example, we might wish to add the following:

instance Genetic Char where type Sequence Char $=$ [Word8] ...

Note that the type signatures of put and get reference Sequence, so in theory the compiler would always be able to determine which function instance (the [Bool] or the [Word8] version) to call. However, the current implementation of type families does not permit duplicate instance declarations. One way to achieve a similar result is to create newtype "wrappers" for each instance declarations, as shown below. 


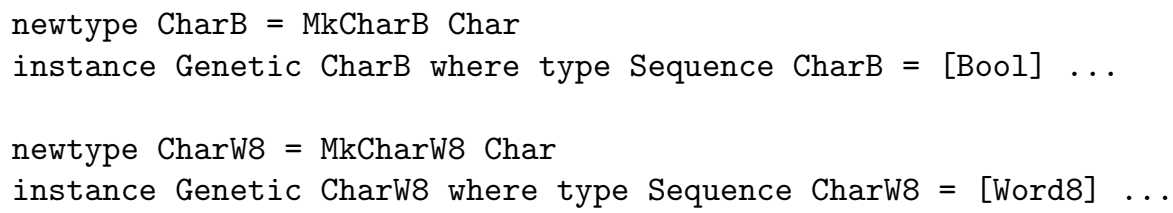

The user can now create new types based on the "wrapped" versions of base types, and automatically derive instances for them, as shown below.

\section{data MyType = MyType $\operatorname{CharB} \ldots$.. deriving Generic instance Genetic MyType}

However, suppose the user now wants to change from using [Bool] for encoded gene sequences to [Word8]. Every reference to CharB will have to be changed to Charw8. References to other base types will have to be modified similarly. Worse still, suppose the user wants to use both [Bool] and [Word8] sequences in the same program. They would have to define multiple versions of their types. This situation is not user-friendly.

Another possibility is to use multi-parameter typeclasses, as shown below. However, we felt that multi-parameter typeclasses were less likely to be familiar to our users than type families ${ }^{2}$.

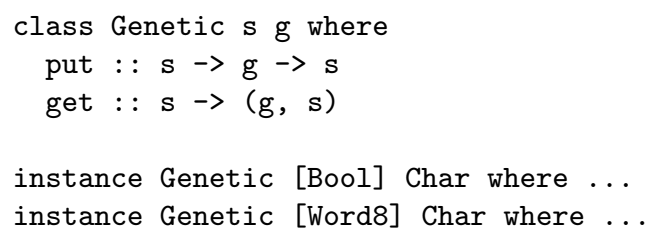

Ultimately we chose to follow the model of commonly-used modules such as Data.ByteString and Data.Map, i.e., having multiple modules that provide the same interface. By simply changing the import statement, the user can change the sequence type. This makes it easy for the user to benchmark different types to determine, for example, whether [Word8] or [Word16] will be more efficient in a given application.

We also provided Reader and Writer monads for operating on an encoded gene sequence. These will be discussed in more detail in Section 7. The final implementation of Genetic is shown below.

\footnotetext{
${ }^{2}$ Hage reported that in 2010, Type Families were enabled in 114 packages on Hackage, and one of the top 10 downloads at that time, while MultiParamTypeClasses were enabled in 321 packages, and nine of the top 10[17]. However, Type Families are a more recent development than MultiParamTypeClasses, and may be overtaking MultiParamTypeClasses in popularity. On 27 June, 2014, we searched stackoverflow (http://stackoverflow.com), a Q\&A forum for programmers, for the tag [haskell] and the term TypeFamilies. This search yielded 81 questions asked or answered during the year to date (409 for all time). A search for [haskell] and MultiParamTypeClasses yielded 55 questions during the year (349 for all time).
} 


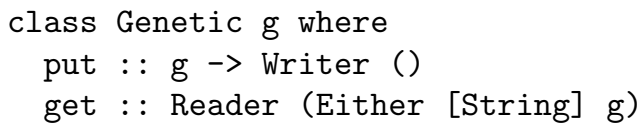

Datatype-generic programming allows Créatúr to automatically generate instances for put and get. The details of how to use datatype-generic programming are described by Magalhães [16] and on the Haskell wiki[18]. Here we will summarise the steps we took to allow implementations of the Genetic class to be automatically generated.

- Implementing Genetic for a set of base types Bool, Char, Word8 and Word16, along with types of the form [a], Maybe a, (a, b) and Either a b, where $\mathrm{a}$ and $\mathrm{b}$ are themselves instances of Genetic.

- Creating a new class, GGenetic, which handles encoding and decoding of the sum-of-products representation of a value.

- Implementing GGenetic for each of the types used in the sum-of-products representation.

- Providing a default implementation of put and get in the Genetic class; they simply invoke the corresponding methods in the GGenetic class.

As a result, the end user can automatically create an instance of Genetic for any type without writing an implementation for put or get, as long as the type is constructed using only the supported base types. For example, we can modify the FlowerColour type to use the automatically-generated genetic encoding scheme by using the language pragma DeriveGeneric, importing GHC.Generics, and declaring FlowerColour to be an instance of Genetic. Now get and put can be used with the FlowerColour type.

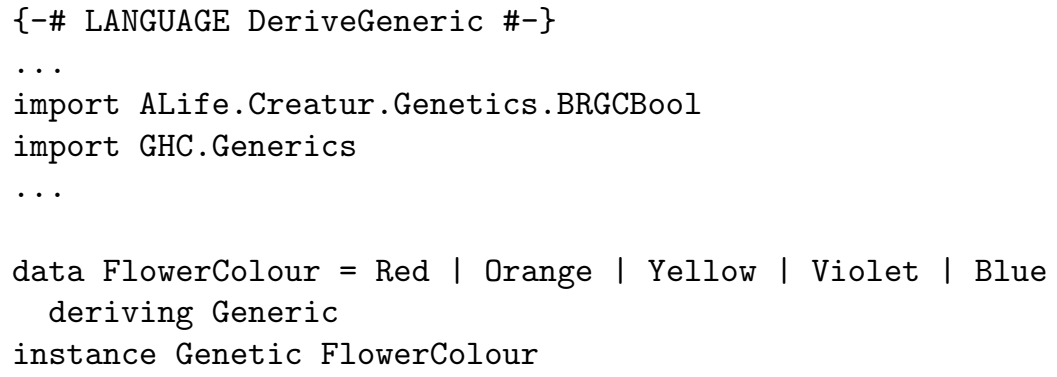

There are three variants of Genetic. The one in ALife.Creatur. Genetics. Code.BRGCBool encodes genes to produce a sequence of Bools. This is practical when the genes of an agent have a small set of possible values. If an agent has genes with a larger number of possible values, it may be better to store their genetic information as a string of numbers. ALife.Creatur.Genetics. Code.BRGCWord8 encodes genes to produce a string of Word8s. Similarly, ALife. Creatur.Genetics. Code.BRGCWord16 uses Word16s.

All three implementations encode integral and character values using a binary-reflected Gray code (BRGC). A Gray code maps values to codes in 
a way that guarantees that the codes for two consecutive values will differ by only one bit[19]. This feature is useful for encoding genes because the result of a crossover operation will be similar to the inputs. This helps to ensure that offspring are similar to their parents, as any radical changes from one generation to the next are the result of mutation alone.

\section{Reproduction}

Recall that in our Plant example, each agent has a single strand of genetic information. During reproduction, the strands from two parents are recombined, creating genetic information for potential offspring. Thus, each parent contributes approximately half of its genetic information to the offspring. The recombination process will be discussed in Section 5 .

Créatúr provides the Reproductive class in the ALife.Creatur.Genetics . Reproduction.SimplifiedSexual module for this purpose. This class can be used with either BRGCBool, BRGCWord8 or BRGCWord16, and contains three functions. The function recombine recombines the genetic information from two potential parent agents, as discussed above. The user must provide the implementation for recombine using a domain-specific embedded language (DSEL) which will be described in Section 5. The function build constructs an agent from a strand of genetic information, if it is possible to do so (i.e. if the genes translate to a valid agent). The user must provide an implementation of this function as well; this is discussed in Section 7. Finally, the make0ffspring function takes two agents and attempts to produce offspring. A default implementation is provided, which calls recombine to create a genome for the child and calls build to construct the child. The definition of Reproductive is shown below.

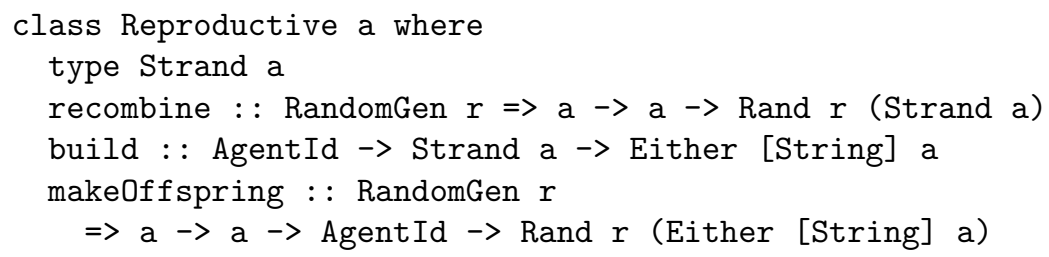

In our Bug example, each agent has two strands of genetic information. During reproduction, the two strands from one parent are recombined to produce two new strands. (The recombination process will be discussed in Section 5.) One of these strands is chosen at random to become that parent's contribution to the child's genome. This is analogous to the production of a gamete (ovum or sperm) in biology. The process is repeated for the other parent. Thus the child has two strands of genetic information, one contributed by each parent. As before, each parent contributes approximately half of its genetic information to the offspring.

Créatúr provides a class for this, also called Reproductive, in the ALife. Creatur.Genetics.Reproduction. Sexual module. As before, this class can be used with either of the encoding methods described in Section 3, and contains 
three functions. The produceGamete function recombines the twin strands of genetic information from two potential parents, using the technique described above. The user must provide the implementation for recombine using the DSEL described in Section 5. The function build constructs an agent from two strand of genetic information, if possible. The user must provide an implementation of this function; this will be discussed in Section 7 .

Finally, the makeOffspring function takes two agents and attempts to produce offspring. A default implementation is provided, which calls produceGamete to produce a single strand of genetic information from each parent, pairs the two strands to create a genome for the child, and calls build to construct the child.

The definition of Reproductive is shown below.

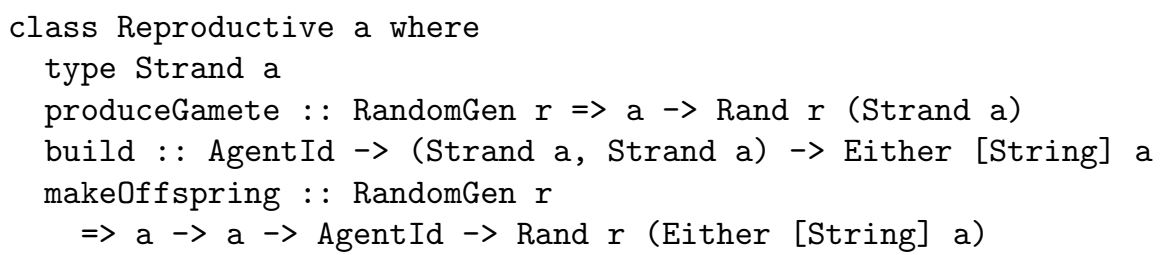

\section{Gene Recombination}

Both of the scenarios described in Section 4 involve shuffling a pair of sequences to produce two new pairs, and possibly discarding one of the sequences. Additionally, occasional random mutations are allowed. The ALife.Creatur. Genetics . Recombination module in the Créatúr library provides a DSEL for genetic recombination. These operations can be applied with specified probabilities and combined in various ways. Two common operations are crossover and cut-andsplice. In crossover (Figure 1), a single crossover point is chosen. All data beyond that point is swapped between strings. In cut-and-splice (Figure 2), two points are chosen, one on each string. This generally results in two strings of unequal length.
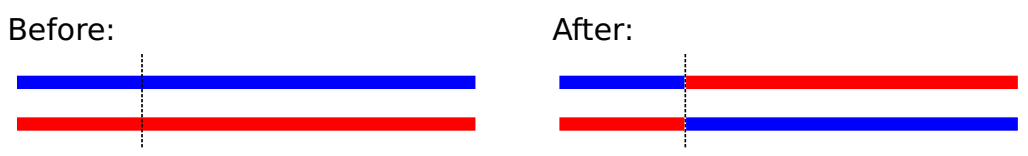

Fig. 1. Crossover

Here's a sample program that might be used to shuffle two sequences of genetic material.

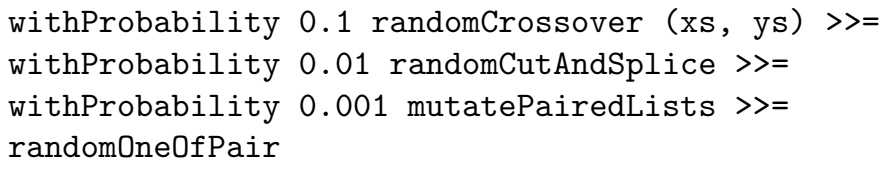




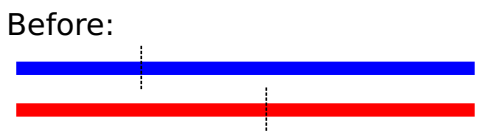

Fig. 2. Cut-and-splice

To illustrate how this program would work, suppose this program acted on the following pair of sequences:

$$
\text { ( }[A, A, A, A, A, A, A, A, A, A],[C, C, C, C, C, C, C, C, C, C])
$$

The first line of the program has a $10 \%$ probability of performing a simple crossover at a random location, perhaps resulting in:

$$
\text { ( }[A, A, A, A, A, A, A, C, C, C],[C, C, C, C, C, C, C, A, A, A])
$$

The second line of the program has a $1 \%$ probability of performing a cut-andsplice, perhaps resulting in:

$$
\text { ( }[A, A, A, A, C, A, A, A],[C, C, C, C, C, C, A, A, A, C, C, C])
$$

The third line of the program has a $0.1 \%$ probability of mutating one or both sequences, perhaps resulting in

$$
([T, A, A, A, C, A, A, A],[C, C, C, C, C, C, A, A, C, C, C, C])
$$

After the first three operations, we have two new sequences. In this example, we only want one of the sequences, so the final line randomly chooses one.

To perform more than one crossover, the operation can simply be repeated. Alternatively, we can choose the number of crossover operations at random. The function repeatWithProbability performs an operation a random number of times, such that the probability of repeating the operation $\mathrm{n}$ times is $p^{n}$. Table 1 contains the full list of available operators.

\section{Gene Expression}

In biology, gene expression is the mechanism that determines the phenotype (the observable traits of the organism) from the genotype (genetic makeup)[20]. Similarly, an ALife species which uses sexual reproduction needs a way to determine the characteristics of an agent from the two strands of genetic information. An agent that arises through sexual reproduction will have two strands of genetic information. When corresponding genes from the two sets are not identical, the dominance rules are applied.

The Diploid class, in the module ALife.Creatur.Genetics.Diploid, represents paired genes or paired instructions for building an agent. Diploid contains the function express. Given two possible forms of a gene or gene sequence, express takes into account any dominance relationship, and returns a gene representing the result. Créatúr uses datatype-generic programming (discussed in Section 3) to provide a default implementation of Diploid, including express. The definition of Diploid is shown below. 
Table 1. The Recombination DSEL

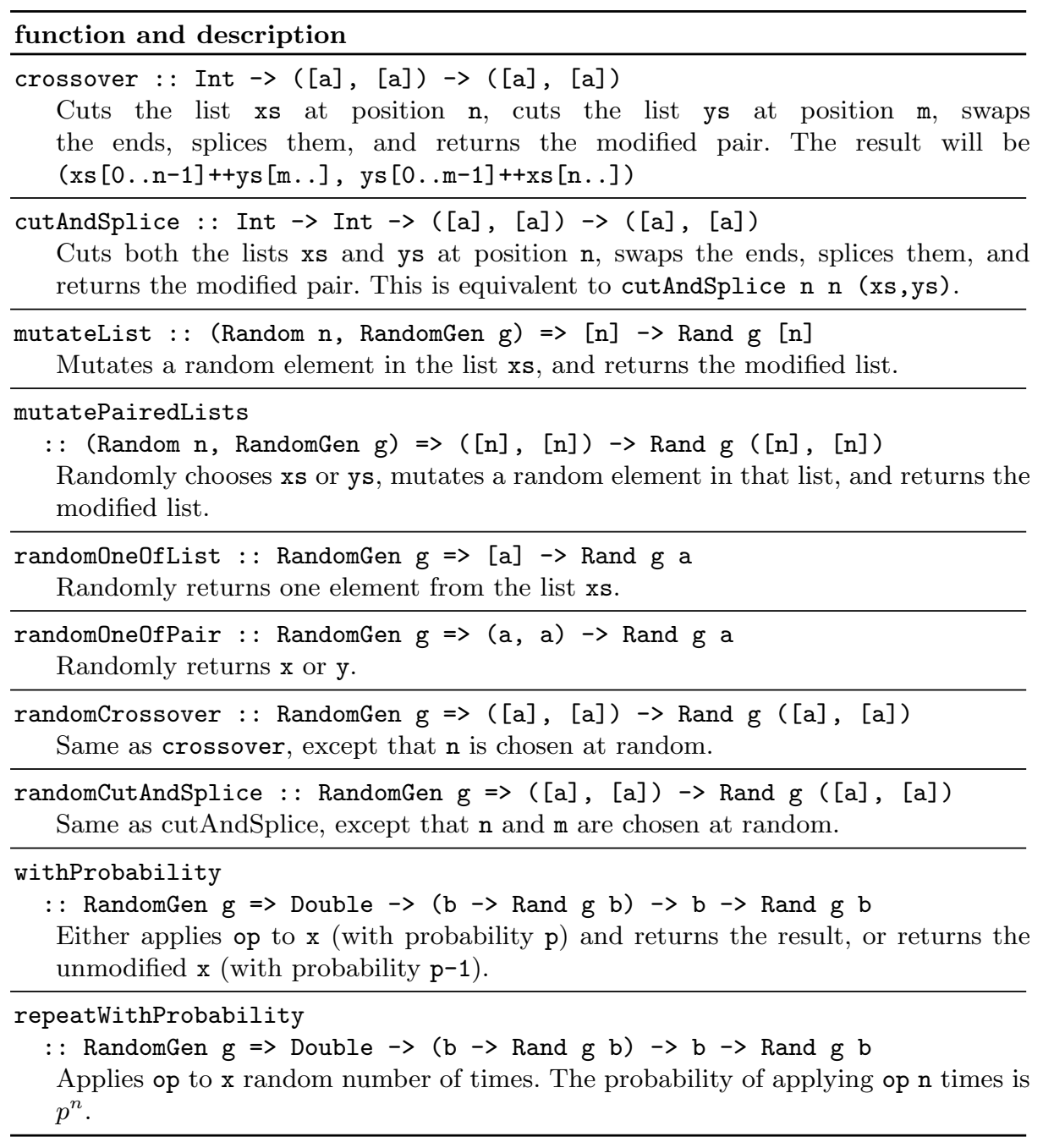

\section{class Diploid g where \\ express : : $g \rightarrow g \rightarrow g$}

Default implementations of Diploid are provided for the following types: Bool, Char, Double, Int, Word, Word8, Word16, Word32, and Word64, along with sequences, tuples, and sums or products of any types that themselves implement Genetic. In practice, this means that the user can often create an instance of Diploid without writing an implementation for express.

In the default implementation of express "small" is dominant over "large". If arrays are of different lengths, the result will be as long as the shorter array. 
express $[1,2,3,4][5,6,7,8,9] \rightarrow[1,2,3,4]$

Consider the following type:

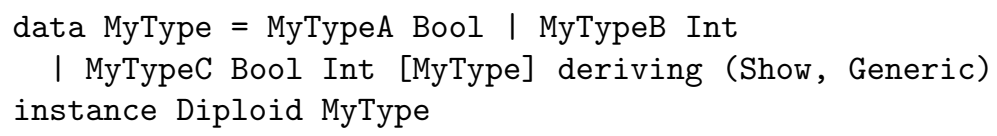

Here are some examples of how express operates.

$\begin{array}{lll}\text { express (MyTypeA True) } & \text { (MyTypeA False) } & \rightarrow \text { MyTypeA True } \\ \text { express (MyTypeB 2048) (MyTypeB 36) } & \rightarrow \text { MyTypeB } 36\end{array}$

When a type has multiple constructors, the constructors that appear earlier in the definition are dominant over those that appear later. For example:

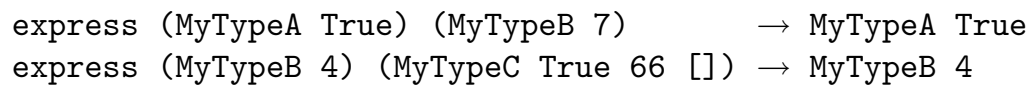

Even with complex data structures, the implementation should just "do the right thing".

Given a numeric type, it would seem that the logical way to express two values is to average them. So why do we instead use the smaller value? In our research with ALife, numeric genes usually control the resources used by an agent. Examples include a gene which specifies the number of neural connections in the agent's brain, or a gene which controls the age at which offspring become mature and are no longer dependent on a parent. Choosing the smaller number helps to ensure that agents use resources efficiently. Of course, a different dominance rule can be used by writing a custom implementation of express.

\section{Constructing an Agent from Its Genome}

Monads "provide a convenient framework for simulating effects found in other languages, such as global state, exception handling, output, or non-determinism" [21]. Since a monad defines a small set of operations that can be used within it, it is essentially a DSEL. Hudak calls monads used in this way "modular monadic interpreters" because they allow different language features to be isolated and given context-specific interpretations, and combined like "building blocks"[22]. In this section we will demonstrate how we use monads to create tools for constructing agents.

As mentioned in Section 4, implementations of the class Reproductive must implement the function build, which constructs an agent from a genome, if the genome is valid. We are now ready to show how this is done.

Recall the definition of Plant from Section 1. To create a plant, we need to determine the flower colour from the genome, and set the ID and energy. The BRGCBool, BRGCWord8 and BRGCWord16 modules define a monad called Reader (unrelated to Control.Monad.Reader), which provides functions for decoding a 
Table 2. The Reader DSEL

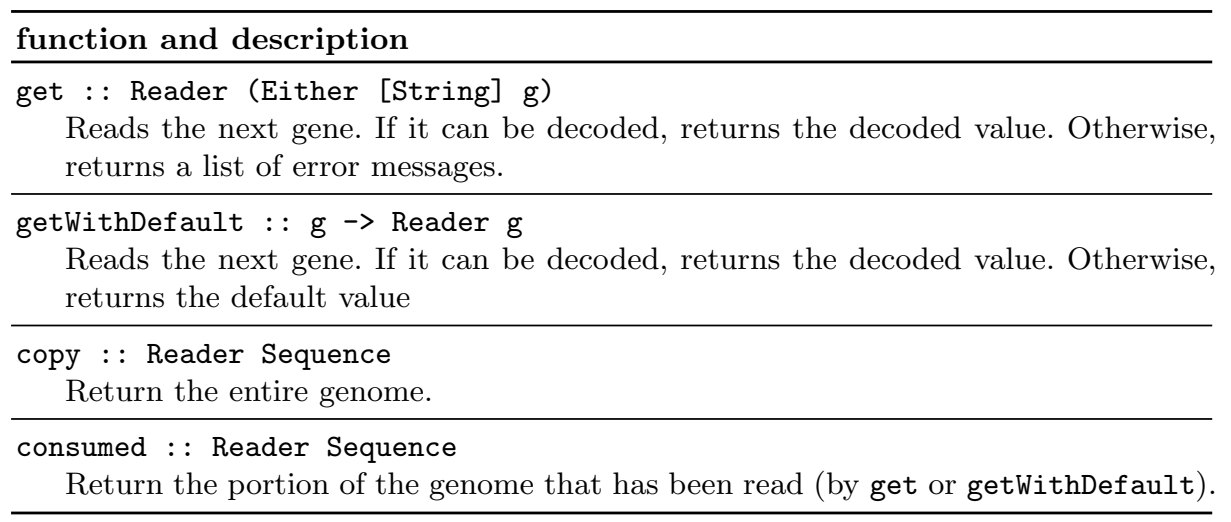

strand of genetic information. Thus, the Reader monad is a DSEL for reading genomes; this language is defined in Table 2.

We can write a buildPlant method using this DSEL. The function will take a String (a unique identifier of the plant to be created), and it will return a program that runs in the Reader monad. That program will return a either a list of Strings containing error messages, or a plant. Thus, the type signature for the buildPlant function is:

buildPlant : : String $\rightarrow$ Reader (Either [String] Plant)

Now to write the program. First, each plant needs a copy of its genome in order to produce offspring; we can use the copy function to obtain this. Next, we determine the colour of the plant. We could use the method get, which returns a Maybe value containing the next gene in a sequence. But consider that our sequence of Bools may not be a valid code for any colour. If an error occurs, we could treat the mutation as non-viable and return Nothing. However, in this example, we wish to create a plant no matter what errors are in the genome, so we will use getWithDefault, with Red as the default value. All plants start life with an energy of 10 . Here is the program:

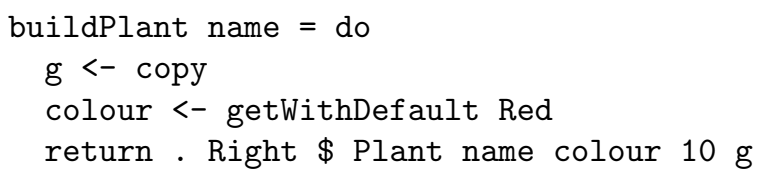

Now, buildPlant is a function that returns a program that runs in the Reader monad. How do we run that program? ALife.Creatur.Genetics. BRGCBool, ALife.Creatur.Genetics.BRGCWord8 and ALife.Creatur. Genetics.BRGCWord16 provide a function for this purpose, called runReader. Now we have everything we need to declare Plant to be an instance of Reproductive. 
Table 3. The DiploidReader DSEL

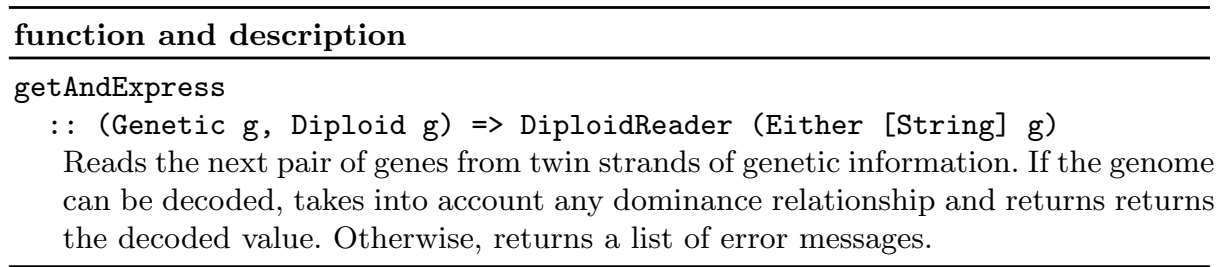

getAndExpressWithDefault : : (Genetic $g$, Diploid $g$ ) $\Rightarrow g \rightarrow$ DiploidReader $g$ Reads the next pair of genes from twin strands of genetic information. If the genome can be decoded, takes into account any dominance relationship and returns returns the decoded value. Otherwise, returns the default value

copy2 : : DiploidReader DiploidSequence

Returns the entire genome (both strands).

consumed2 :: DiploidReader DiploidSequence

Returns the portion of each strand that has been read (by get or getWithDefault).

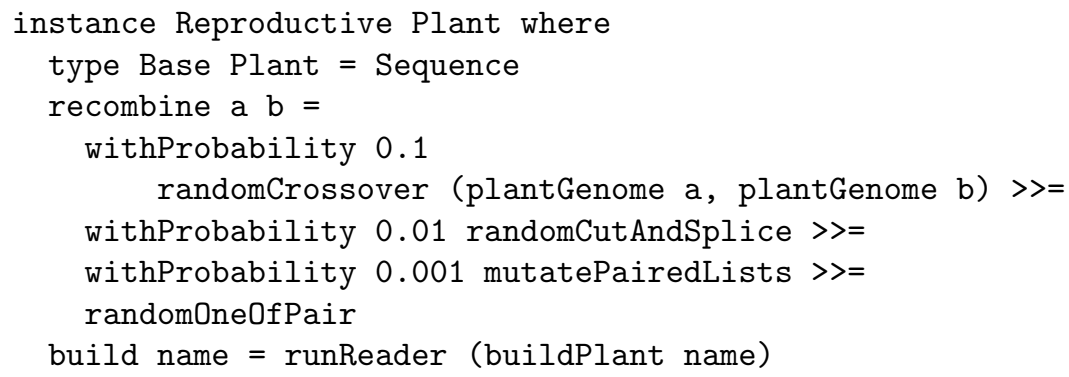

Recall the definition of Bug from Section 1. Now we have two strands of genetic information which determine the bug's traits. The BRGCBool, BRGCWord8 and BRGCWord16 modules define a monad called DiploidReader for this situation. The DiploidReader monad is also DSEL; this language is defined in Table 3 .

Our buildBug method will take a String (a unique identifier), and it will return a program that runs in the DiploidReader monad. The implementation is similar to buildPlant, except that the single-strand operations have been replaced with versions that work with both strands.

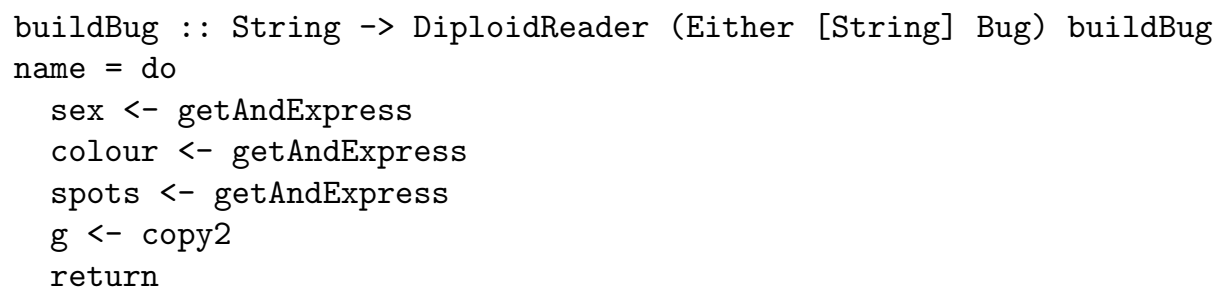

$\$$ Bug name $\langle \$\rangle$ sex $\langle *\rangle$ colour $\langle *\rangle$ spots $\langle *\rangle$ pure $10\langle *\rangle$ pure $\mathrm{g}$ 
The runDiploidReader function runs a program written in the DiploidReader DSEL and returns the result. Now we can implement Reproductive.

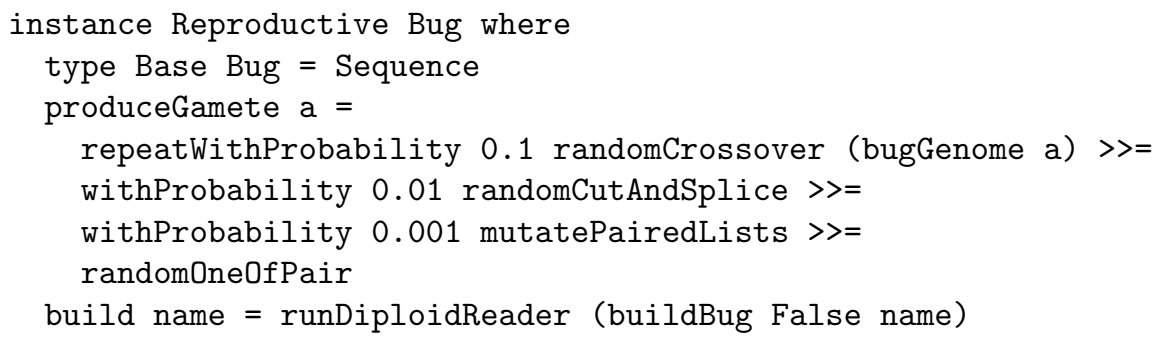

The BRGCBool, BRGCWord8 and BRGCWord16 modules also define a monad called Writer, used for encoding genetic information. This is useful for generating an initial population. The Writer DSEL consists of one function, put, which writes a gene to a sequence.

One approach to creating an initial population is to feed random strings of genetic information into the function that builds the agent, but instruct it to keep only as much of the sequence as it needs to build a complete agent. The functions consumed (from the Reader DSEL) and consumed2 (from the DiploidReader DSEL) are useful here. For example, we can modify the buildBug method from Section 7 to accept a boolean that tells it whether or not to discard the unread portion of the sequences.

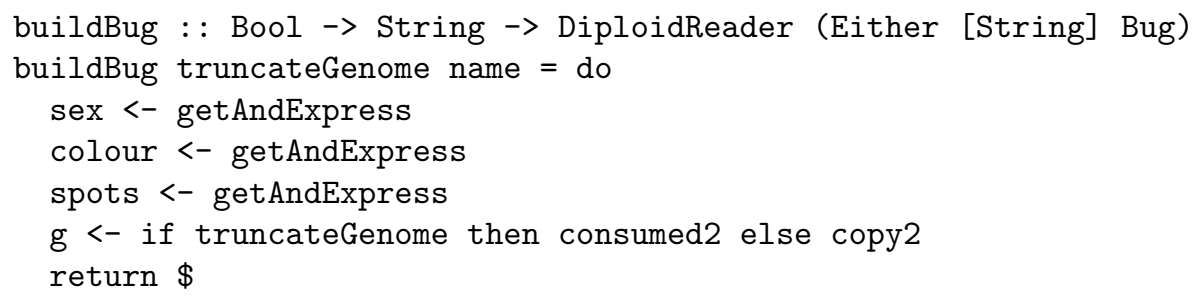

\section{Conclusion}

As library developers, we found it straightforward to use the datatype-generic programming feature of GHC to specify how to derive instances of Genetic and Diploid. The user has it even easier; they can simply declare their custom types to be instances of these classes, taking advantage of the default implementation we provide. Perhaps type families could support duplicate instance declarations in appropriate circumstances, or alternatively, type families and multi-parameter typeclasses were unified into one language feature with a common design.

Each of the DSELs we developed required only a small set of operations; it was easy to embed them in Haskell. In this way we avoided having to design 
a language and write a parser for it. The user does not have to learn a "new" language, and rather than being restricted to the semantics of the DSEL, the user has access to all the features of Haskell, if needed. Finally, using monads for the Reader, DiploidReader and Writer DSELs allowed us to isolate the stateful computations required to read and write genes.

\section{References}

1. Langton, C.G.: Artificial life. In: Langton, C.G. (ed.) Artificial Life: The Proceedings of an Interdisciplinary Workshop on the Synthesis and Simulation of Living Systems, Held in September, 1987, in Los Alamos, New Mexico, pp. 1-48. AddisonWesley, Redwood City (1989)

2. Cao, L.: Data Mining and Multi-agent Integration. Springer, Boston (2009). http:// dx.doi.org/10.1007/978-1-4419-0522-2

3. Miranda, E.R.: A-life for music: music and computer models of living systems. A-R Editions, Middleton (2011). http://www.worldcat.org/search?qt=worldcat_ org_all\&q=9780895796738

4. Dessalegne, T., Nicklow, J.: Artificial Life Algorithm for Management of Multireservoir River Systems. Water Resources Management 26(5), 1125-1141 (2012). http://dx.doi.org/10.1007/s11269-011-9950-7

5. Dennett, D.C.: Consciousness explained. Penguin (1993). http://www.worldcat. org/isbn/9780140128673

6. Fuller, S., Wolpert, L.: Transcript of the debate between Professor Steve Fuller and Professor Lewis Wolpert at Royal Holloway College (February 2007). http://www.bcseweb.org.uk/index.php/Main/RoyalHollowayCollegeDebate (cited July 03, 2010, 01:11:46)

7. Mitchell, M.: An introduction to genetic algorithms, 2nd edn. Prentice Hall of India, New Delhi (2002)

8. de Buitléir, A., Russell, M., Daly, M.: Wains: A pattern-seeking artificial life species. Artificial Life 18(4), 399-423 (2012)

9. de Buitléir, A., Russell, M., Daly, M.: A Functional Approach to Neural Networks. The MonadReader 21, 5-24 (2013). http://themonadreader.files.wordpress.com/ 2013/03/issue21.pdf

10. de Buitléir, A.: Créatúr GitHub. GitHub repository (2014).https://github.com/ mhwombat/creatur

11. de Buitléir, A.: Créatúr Tutorial (2014). https://github.com/mhwombat/ creatur-examples/raw/master/Tutorial.pdf

12. Peyton-Jones, S.: Haskell 98 language and libraries: the revised report. Cambridge University Press, Cambridge (2003). http://www.worldcat.org/isbn/ 9780521826143

13. Lämmel, R., Jones, S.P.: Scrap your boilerplate: a practical design pattern for generic programming. In: Shao, Z., Lee, P. (eds.) In: Proceedings of the 2003 ACM SIGPLAN International Workshop on Types in Languages Design and Implementation, TLDI 2003, pp. 26-37. ACM, New York (2003). http://doi.acm.org/10. $1145 / 604174.604179$

14. Lämmel, R., Jones, S.P.: Scrap more boilerplate: reflection, zips, and generalised casts. In: Proceedings of the Ninth ACM SIGPLAN International Conference on Functional Programming, ICFP 2004, pp. 244-255. ACM, New York (2004). http://doi.acm.org/10.1145/1016850.1016883 
15. Lämmel, R., Jones, S.P.: Scrap your boilerplate with class: extensible generic functions. In: Proceedings of the Tenth ACM SIGPLAN International Conference on Functional Programming, ICFP 2005, pp. 204-215. ACM, New York (2005). http://doi.acm.org/10.1145/1086365.1086391

16. Magalhães, J.P., Dijkstra, A., Jeuring, J., Löh, A.: A generic deriving mechanism for haskell. In: Proceedings of the Third ACM Haskell Symposium on Haskell, Haskell 2010, pp. 37-48. ACM, New York (2010). http://doi.acm.org/10.1145/ 1863523.1863529

17. Hage, J.: DOMain Specific Type Error Diagnosis (DOMSTED). Technical Report UU-CS-2014-019 (2014). http://www.computerscience.nl/research/techreps/repo/ CS-2014/2014-019.pdf

18. contributors W. GHC. Generics (2013). Wiki page. http://www.haskell.org/ haskellwiki/GHC.Generics

19. Gray, F.: Pulse code communication. Google Patents. US Patent 2,632,058 (1953). http://www.google.com/patents/US2632058

20. Lewontin, R.: The genotype/phenotype distinction. In: Zalta, E.N. (ed.) The Stanford Encyclopedia of Philosophy (Summer 2011)

21. Wadler, P.: Monads for functional programming. In: Jeuring, J., Meijer, E. (eds.) AFP 1995. LNCS, vol. 925, pp. 24-52. Springer, Heidelberg (1995). http:// homepages.inf.ed.ac.uk/wadler/papers/marktoberdorf/baastad.pdf

22. Hudak, P.: Building domain-specific embedded languages. ACM Computing Surveys (CSUR) 28(4es), 196 (1996) 